\title{
Ternary Classification of Product Based Reviews: Survey, Open Issues and New Approach for Sentiment Analysis

\author{
Sushila Sonare, Megha Kamble
}

\begin{abstract}
Now-a-days, it is very common that the customers share their thoughts about any product, brand and their experience in social media. The analysts collect these reviews and process it, to extract meaningful information about the product. The beauty of social media is, it's involved in all the domains. So the analysts got reviews from different social media and platforms for almost all kind of thing. The Sentiment Analysis is applied to predict outcomes for getting useful information, for ex.; like predict the blockbuster for a movie, rating for any new launches and many more. This type of prediction is really helpful for the customer to buy any goods or take any services in this competitive world. This paper is focused on e-commerce website reviews which are normally in text form with some special characters and some symbols (emojis). Each word in this text set got some meaning in terms of context, emotion and prior experience. These characteristics contribute to some of the features of text data for prediction. The objective of this paper is to compile existing research works on text analysis and emotion based analysis. The open issues and challenges of document based sentiment analysis are also discussed. The paper concluded with proposing a new approach of multi class classification. Ternary classification for classes positive, negative and neutral is suggested primarily for product based text and emoji reviews on Twitter social media.
\end{abstract}

Keywords: Sentiment Analysis, reviews, machine learning, e-commerce, Real time.

\section{INTRODUCTION}

The Sentiment Analysis is one of the Natural Language Processing (NLP) techniques that used to find opinion, whether data is positive, negative or neutral. Sentiment Analysis worked on textual data for businesses monitor like brand and product based sentiment that calculated from customer feedback, needs of customer.

Sentiment analysis is mainly based on attitude, emotion or experience. First step in sentiment analysis is to collect the opinions for a particular product from the customers. Different users or customers have different opinions about the same product. Also ensure to collect opinions from different

Manuscript received on 15 March 2021 | Revised Manuscript received on 23 March 2021 | Manuscript Accepted on 15 April 2021 | Manuscript published on 30 April 2021.

* Correspondence Author

Sushila Sonare*, Department of Computer Science and Eengineering, Lakshmi Narain College of Technology, University, Bhopal, India. Email sushila09s@gmail.com

Dr. Megha Kamble, Department of Computer Science and Engineering, Lakshmi Narain College of Technology, University, Bhopal, India. Email: meghak@lnct.ac.in

(c) The Authors. Published by Lattice Science Publication (LSP). This is an open access article under the CC-BY-NC-ND license (http://creativecommons.org/licenses/by-nc-nd/4.0/) platforms so it will give you a clear image and all dimensions about any product. This paper considers twitter as a social media platform to collect these opinions, primary cause is it is popular these days and second cause is, as it limits the review to 140 characters, it is precise and can be useful for effective analysis.

Customers' opinion is really helpful for other customer as well as seller. To know the information about any product, customer can get best product in available amount as well as quality. This kind of opinion also useful for seller, they can know what was good and what was bad, regarding to their product so they can maintain its stock and quality for profitable business. The reviews are mostly in text form, which is unstructured. This information contains some general words that can classify our opinion into different classes. In multi-class sentiment classification opinions are divided into strong positive, strong negative, positive, negative and neutral [9]. Sentiment context like good, best, super, excellent etc categorized as strong positive opinion. Same way, bad, worse, not good, unhappy, disgusting etc words indicate negative impact of product which is categorized as strong negative opinion. The main problem arises when opinions take conjunction form where customers are writing two sentences connected by "and", "or", "but" connectors. There might be one part as positive with respect to some feature but other part negative with respect to some another feature. Now with such reviews, there is ambiguity of considering them as positive or negative. Reviews that do not clearly mention any opinion, that are treated as neutral opinion [3].

Sometime customers use some special symbols to write review, these symbols have specific meaning. These symbols are to be converted into text, for processing and then classification into particular opinion class is done and in turn these reviews generate accurate prediction [4].

\section{Overview of Machine Learning}

Machine Learning methods are mainly used in sentimental analysis. As shown in Fig-01, machine learning consists of a set of algorithm that can be applied for social media text data for sentiment analysis. Next few paragraphs are listing some important algorithms of machine learning in this context.

A. Supervised Learning - In this method, the dataset, trained the machine with the help of data which is already labeled, so the new data will mapped with input and output is more accurate.

Main goal of this, provide training to machine learn "how to map input to the output". 

Analysis

Suppose we need a machine that helps to predict a path from current location to desired location. To develop such type of supervised machine, first required training set. This training set contains all features like weather condition, time of day, route chosen, working day/ weekend etc. So that machine take direct relationship between given features like weather (Is raining time?) and time of day, route and time of day, working day/weekend and route etc [9].

\section{Types of supervised leaning:}

- Regression: Regression is used when any problem statement based on finding amount of a participation feature. In this type of problem be solved by regression. The main advantage of this method is, finding the continuous value of feature. This value is always probabilistic interpretation and the algorithm should avoid over fitting.

Based on above scenario if machine will able to find suitable route for particular time, it might take time as main feature to find suitable solution. That means which path take minimum time to travel (it taken amount of time) come under regression.

○ Linear regression

- Logistic regression

- Polynomial regression

○ Stepwise regression

- Lasso regression

- ElasticNet regression

- Classification: classification is divide or group the output data into classes. That means same type of output belongs to a same class.

Based on above scenario, if machine will able to find route that based on traffic. A particular route may have less traffic of more traffic, only two cases possible. This problem belongs to two classes one less traffic and second one, more traffic. This kind of problem comes under classification. The outputs classified into classes.

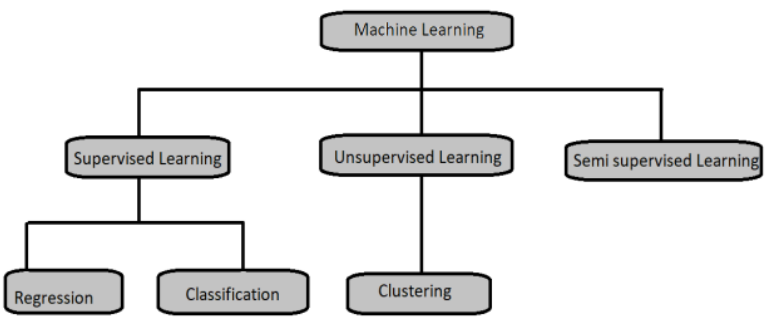

Fig. 1.Classification of Machine Learning

B. Unsupervised Learning - In this method, the machine is trained with data which is not labeled. In this scenario machine has to learn itself. Unsupervised learning is neither classified nor labeled, it learn based on own experience. This is used for more complex tasks [9].Take an example, a baby and his family dog. Baby knows about dog, it has 2 eyes, 2 ears, 4 legs that walk on it. Now suppose in that house another pet (cat) came. For a baby it also seems like dog because it has same feature that he learned previous. Based on his knowledge it comes under dog category.Unsupervised learning helps to find useful feature for categorized test data into proper category. This technique provides unlabeled data to label from self learning model. That means machine itself find some pattern from given data set, to find a solution for the given problem.

\section{Types of unsupervised learning:}

- Clustering: This technique based on grouping the unlabeled data. That means similar types of data are belonging into a group (cluster). It's clearly says that, in group all data have a similarly properties and they are different from other group. This technique mainly used in such a place like, image processing in biology research for pattern, text data based on contain is organized into topic hierarchy, spam filtering, criminal activity, sports etc. Some of famous cluster are:

○ K-Mean clustering

○ Mean- shift clustering

○ K-NN(k nearest neighbor)

○ Hierarchical clustering

- Probabilistic clustering

- Association Rules: this technique based on rule base approach. According to this method finding relationship between variables that are present into given dataset. This method frequently used all relationship that is present in dataset. Some of popular association techniques are:

○ Apriori algorithm

○ FP-Growth Algorithm

○ Dimensionality reduction

C. Semi-supervised Learning - This type of learning used, trained unlabeled data with the help of labeled data for testing. The main task of semi-supervised approached is to label large amount of unlabeled data through labeled data which is small in size [9].

Take an example, a kid has 40 cars (toys) and his elders explain only 4 toys as a car. Now the kid, automatically able to find remaining 36 toys label as a car.

Semi supervised technique has tiny boundaries between supervised and unsupervised. For making this boundary more accurate, it should include some characteristics to learning datasets:

- Size of unlabeled portion: the size of unlabeled must be large enough, that means label data $4-5 \%$ of actual data set. If label data size increased then it come under supervised learning.

- Input-Output proximity: take unlabeled input and tries to find output based on labeled data proximity. All the same type of data must be close proximity to each other.

- Simplicity and complexity of labeling: when influence of labeled data is complex then our problem becomes more complex as compare to original.

- Inductive and transductive learning: inductive means to build generic rule for classification then provide test cases to it. Transduction not based on generic rule, its generated reasoning for specific training cases and applied this reasoning to specific test cases for training.

Algorithms are used to semi supervised learning:

○ Self training

- Generative

- Low-density Separation

○ Practical application

Supervised and semi-supervised learning used for, sentiment analysis. This learning techniques classified into binary and multiclass.

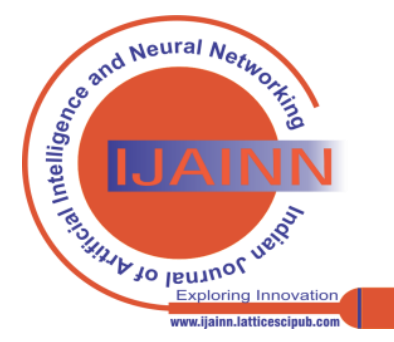


This paper proposes the classification algorithm for three classes of sentiments. The organization of this paper is divided into six parts. Section 1 deals with introduction and problem definition??. Section 2 discusses existing work relating reviews and sentiment analysis from the detailed survey. Section 3 states the design and characteristics of existing methods and proposed method. Section 4 describe in brief about existing / current / latest implementation method associated with the proposed work of sentiment analysis. Section 5 contains comparison with other research, followed by conclusion in section 6 .

\section{RELATED WORK}

This part provides significant and relevant issues in the literature of the paper. There are two most common approaches in text analytics, machine learning and natural language processing. Number of references in the study describe supervised (SML), unsupervised and semi supervised approaches for sentiment analysis.

The efficiency of ML algorithms depend on various factors, data representation is one of the import factors. Different interpretation will interact more or less and various meaning cover behind the data it's depends on understanding factors. Domain specific knowledge used to design depictions, to learn with generalized and search for AI, motivate to create more efficient algorithms.

Sentiment Analysis (SA) mainly categorized in four parts namely Document Based Sentiment Analysis (DBSA), Sentence Based Sentiment Analysis (SBSA), Aspect Based Sentiment Analysis (ABSA) and Comparative Sentence Analysis (CSA).

B. Seetharamulu et al, developed deep learning based framework that classify customer reviews into positive and negative. B. Seetharamulu et. al. used supervised machine learning methodology to build a classifier. It is based on deep learning based sentiment analysis (DL-SA), the goal of them is identify each sentence's sentiment orientation [1].

Deep neural network algorithm mainly learns to interpret data at high level and prevent laborious tasks. This model's success depends on existence of the data regarding large-scale training. This deep learning based model is for emotion recognition (DLER). The model worked on CNN algorithm which is based on neural network as part of deep learning process and train data on prior information so predict suitable result. This model compares DL-SA with existing model used in Weakly Supervised Definition Extraction WDE [1].

Azwa Abdul Aziz et. al., worked on opinion mining based on reviews or feedback from costumers. According to author, in-domain sentiments works perfectly fine but real time data sets model performance might be changed, similarly this SML decrease in cross domain datasets. These model works on Contextual Analysis (CA), it provide relationship between words and sources to be constructed in tree structure that is defined as Hierarchical Knowledge Tree (HKT). Azwa Abdul Aziz et. al. worked on Tree Similarity Index (TSI) and Tree Differences Index (TDI). The main goal is to find performance measure of SML models in real time data then, establish relationship in CA that used to understand the structured knowledge of data [2].

Azwa Abdul Aziz et. al. focus in SML as a text recognizer for data comparison. SML exploits training data for train classifier so that it predicts required result. Classifiers mostly trained set based on n-grams which is contiguous sequence of $\mathrm{n}$ items from given sample text. The concept behind CA is based on text, so it is converted into HKT. HKT is using unlabeled data that helps to understand subject and data knowledge. CA approach is used to predict rate of success or failure of other models in SML. This model uses two processes for text normalization; first stemming and second lemmatization [2].

Stemming works on eliminating affixes ie. suffix, prefix and infix from word's order to obtain stem. Lemmatization process is for grouping the different inflected forms of word so it can be analyzed as single text. Azwa Abdul Aziz et. al. worked on cross-domain replication [2].

Kartikkayini T and N K Srinath, worked on comparative polarity analysis based on Senti algorithm using NLTK and Datumbox tool. Kartikkayini T and N K Srinath analysis worked on three level, first document level, second sentence level and last opinion mining classification based on subjective and objective. Model clearly classified positive polarity, negative polarity and neutral polarity. This model based on raw data and passed it to JSON parser through IDE (RStudio and Python), after processing data, passed it in NLTK for sentiment analysis and finally converts into chart [2].

Senti algorithm takes overall reviews for particular product and divided into sentiment score, partial sentiment score and sentence score. Based on score overall score will maintain as average of partial sentiment score and sentence score [3].

Ronen Feldman, worked on reviews analysis. According to Ronen Feldmen sentiment collect form social media like facebook, message boards, blogs, forums, tweets etc. Ronen Feldman mainly focus on all field of sentiment analysis; document-level sentiment analysis, sentence-level sentiment analysis, Aspect- based sentiment analysis, Comparative sentiment analysis and Sentiment lexicom acquisition.

According to Ronen Feldman corpus of document take as input to system, convert to text then pre processing based on variety of linguistic tools that work on stemming, tokenization, part of speech with tagging, entity extraction and relation extraction. Document analysis module used linguistic resources for annotation pre-process documents. This annotation used in variety of visualization tools [4].

K. Saranya and S. Jayanthy, demonstrated work based on text based sentiment along with emotion. Onto-based sentiment classifications mainly provide emotion to the text. This model takes emotion text as an input that has to be classified into positive opinion or negative opinion. Emotion texts passed to NLP for process and this model analyzed and provide annotation to text [5].

K. Saranya and S. Jayanthy work on movies reviews dataset and categories it positive, negative and neutral. The dataset is created, by including thumbs-up and thumbs-down symbols for classification of sentences.

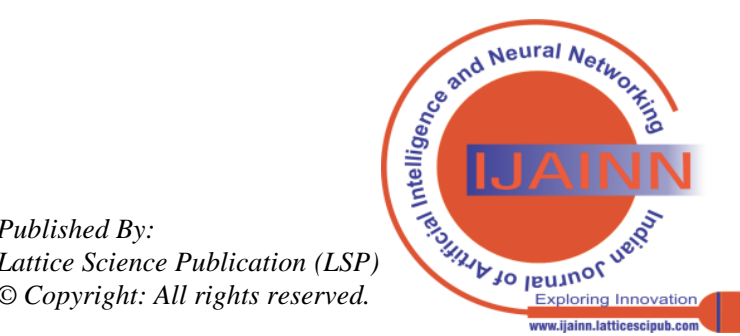


The models identified affective class hierarchy in WordNet so emotions assigned to semantic roles for creating emowords. These emoword used to provide proper emotion hierarchy. For that k. Saranya and S.

Jayanthy used hybrid of SVM and Navie-Bayes algorithms for better result [5].

Nadijim Frechet et al. work on syllabi that are selected from top 50 universities ranked by QS World University Ranking list. This model used 1000 unique sources and creates indexing, to know utilization of publication that is form of citation. So it can classify the data in proper fields using state-of-the-art machine learning models. Also conceder, extracted text data from audio and video so understand Wordscores [6].

Nadijim Frechet et al mainly compare $\mathrm{R}$ and Python and found for large-scale or prediction based application is more suitable in Python. Their study included various text-analysis packets like tidyverse, tidytext, stm and quanteda in $\mathrm{R}$ and pandas, NumPy, Scikit-learn, NLTK, spaCy and Gensim in Python [6].

K Sudheer and Dr. B Valarmathi works on customers reviews from twitter, its real time sentiment analysis. Try to collect reviews from e-commerce sites and used machine learning algorithms to label it. This task basically focuses on sentiment lexicons, sentiment analysis and machine learning models [7].

K Sudheer and Dr. B Valarmathi, used twitter streaming API for downloading live tweets. Tweets collected from amazon 50000, e-bey 25000 and alibaba 25000. In this data remove retweets, urls, \# tag, and special characters. This model used NLTK libraries for stemming, tokenization and machine learning algorithms, parsing and semantic reasoning. This worked more than 50 corpora and lexical resources for sentiment analysis [7].

This model worked on Document Frequency (DF) for finding frequent words in data based on predefined threshold range. In this model compares Naïve Bayesian classification, max-entropy classification, and decision rule based classification [7].

Lijuan Huang et al., worked on polymerization topic sentiment model (PTSM). PTSM model extract data and filter it so get sentiment information from online reviews. This model proposed hidden sentiment information in document-level. In this process filter unnecessary information from online reviews as well as remove over-fitting problem so it's easy to apply machine learning algorithm on it to get accurate prediction from it [8].

Lijuan Huang et al., first established data dictionary based on corpus method and lexicon based approach. This data dictionary used manual information to find most positive relevant and most negative relevant features and ranked it two groups by using of word segmentation (WS). After creating dictionary forward it to neural network (NN) and support vector machine (SVM) then passed to PTSM to get more accurate prediction as compare to existing methods [8].

Abinash Tripathy et al., works on text reviews to find the prediction of products for that in this model used hybrid machine learning algorithm. First this model work on SVM algorithm and generate output from it, now pass it as input for artificial neural network (ANN) algorithm for sentiment classification. This model worked on Term frequency-inverse document frequency (TF-IDF) and CountVectorization (CV) method to find feature by using IMDb data set and polarity dataset [9].

In this model TF-IDF value increase when frequency of word increased in document. Term frequency means numbers of time the word repeated in text where as inverse document frequency the occurrence of term in whole document. CountVectorization convert text document to sparse matrix based on token counts [9]. This model takes input as two movies reviews datasets IMDB and polarity, then applied $\mathrm{k}$-fold cross validation approach. Before that preprocess data to remove stop words like numerical values, special character, url and html tags. After that preprocess text reviews to convert numerical vectors by $\mathrm{CV}$ and TF-TDF method then pass this data to SVM to calculate sentiment score. Provide this score to ANN for classification as positive and negative polarity [9]. Jaspreet Singh and Gurvinder Singh, worked on aspect based sentiment analysis technique. Take reviews from user and preprocess for removal stop-words, tagging POS then performed NLP and ML techniques to identify QoS features and logistic regression to improve QoS for realistic data set. This model worked on statistic method for analyzing user reviews to set positive and negative values. Logistic regression generate coefficient formula for calculate probability of QoS features [10]. Jaspreet Singh and Gurvinder Singh used Support vector machine (SVM) and Gradient boosting decision tree (GBDT) for sentiment analysis and found SVM worked fine when data is simple structure but if database is sparse then it not working properly. GBDT worked fine for complex database but facing over fit problem [10].

Jaspreet Singh and Gurvinder Singh works on post process method. This model take input as pre process file then tagging POS, this text files loader (in python 3.6) using UTF-8 encoding of reviews. This encoded token pass to class assignment for labeling it for finding QoS features. This model take web services reviews like amazon cloud, amazon antivirus products and amazon software services [10].

Jeong Woong Sohn and Jin Ki Kim, worked on social network service (SNS) that is on web based services to help consumers to shear their experience and thought to their connection lists. Consumers see and share other consumers' opinions and interest related to any product in various platforms like product reviews, blogs, and SNSes. This model based on word-of-mouth concept [11].

SNSes, mainly deal with all web sites that is related to product and studies whole sites to assess reliability, trust and web credibility. SNSes provide the function of content shearing, as well as identity management with context awareness.

These services provided reliability, ecommerce price and cost that are important feature to online shopping decisions. Mainly social commerce features based on economy, necessity, reliability, interaction and sales promotion [11]. Jeong Woong Sohn and Jim Ki Kim, model takes attributes from shopping malls, homepage information, SNS functions and social commerce features included then extract social commerce attributes that define purchase intention [11].

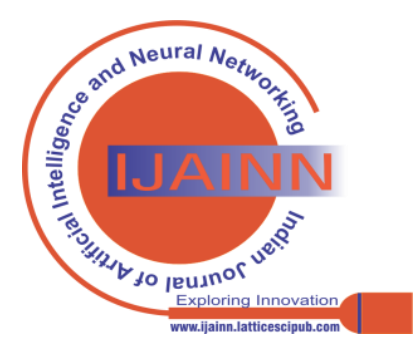


Valerio Basile et al., worked on SENTIPOLC (SENTIment POLarity Classification Task) using Italian tweets, based on EVALITA evaluation campaign. This model on NLP to detect whether tweets is subjective, polarity or irony. This model mainly found tweets subjective or objective so classified accordingly. Similarly found polarity, based on it classified it positive, negative, neutral or mixed sentiment. Last one, irony detection, whether message is ironic or not. This ironic messages lead to wrong polarity. These messages add annotation as literal polarity that shown potential used of system as well as observe patterns of irony [12].

Valerio Basile et al., compares with previous gold standard SENTIPOLC that is 2014 and 2016 to current gold standard SENTIPLOC in 2018 data sets and their outcomes. This model cover most popular algorithm like SVM, Navie Bayes, K-Nearest Neighbors and Decision Trees. This model takes SentiWordNet in AFINN, HU-Liu Lexicon, Whissel's Dictionary as native tools for Italian that is preprocessing like tokenisers, POS tagging and parsers. This model encodes massages in word-based then syntactic and semantic features added with included micro blogging such as emoticons and hashtags. ColingLab, INGEEOTEC and CoMoDi used for that. This model also worked on deep learning methods like UniPI and SwissCheese [12].

Valerio Basile et al., after using internal resources this lexicon worked on external resources for sentiment analysis. For SA this model used Samskara, ItaliaNLP, CoLingLab , CoMoDi, Unitor and IRADASE . This model use CNN for labeling with SwissCheese and Unitor [12].

Cornelius Puschmann and Alison Powell, mainly worked on opinion mining based on news paper articles from various news papers: USA Today, The New York Times, The Washington Post, The Guardian and The Daily Telegraph. In this data set also include trade publications such as Advertising Age, Bank Technology News and Institutional Investor [13].

Cornelius Puschmann and Alison Powell, used Linguistic Inquiry and Word Count (LIWC), calculates degree among different peoples that use different categories of words by a text, emails, speeches, poems, translation and transcript speech etc [13].

Cornelius Puschmann and Alison Powell, worked on finding aspect based on sentiment analysis for approximate human judgment. This model mainly, understands perception of sentiment analysis and other analytic methods, to understand social media. This model focus on more public discussion gathering from social media and computational processes to analysis their results interpretation, efficiency and effectively used to predict "user behavior" [13].

Mirsa Karim and Smija Das, worked on sentiment analysis based on; rule based mechanism and machine learning technology. This model takes movie reviews dataset from cornell university which are labeled with positive and negative labels. This model uses rule based mechanism that uses mining algorithms to find the features and opinion for a particular product [14].

Mirsa Karim and Smija Das model, works on Valence Aware Dictionary and Sentiment Reasoner (VADER) that takes the compound score. Based on compound score is more then and equal to 0.5 consider as positive, less then and equal to -0.5 consider as negative and -0.5 to 0.5 score consider as neutral [14].

Mirsa Karim and Smija Das, worked for sentiment

Retrieval Number: 100.1/ijainn.B1008021221

DOI:10.54105/ijainn.B1008.041221

Journal Website: www.ijainn.latticescipub.com analysis. This model took client rating and reviews as input, perform text mining on it for sentiment analysis. This model worked on LDA analysis for NAÏVE BAYES, sentiment VADER and sentiword net techniques for finding the compound score, POS tagging, synset score to build vocabulary and training. The main aim of this model to find more accurate and precise anticipating the conclusion of sentence as well as found sentiment associate with sentence [14].

\section{METHODLOGY}

This Sentiment classification technique divided into two types: binary classification, multi-class classification. Binary sentiment classification, classified document into two labels: positive polarity and negative polarity. Whereas multi-class classified document into multi labels: strongly positive, positive, neutral, negative, strongly negative polarity [9].

Figure-02 showed the generic architecture of Sentiment Analysis. The main objective of this research is takes the reviews from customer regarding to products in form of tweets. This corpus need some preprocess of data like removing stop words, stemming, lemmatizing, chink and chunk so that dataset should be fine data.

After preprocessing provide this dataset to POS tagging with Navie Bayes Method or WordNet with Navie Bayes Method. Now data set is ready to perform machine learning techniques like regression, SVC etc. To improve this model speed, pickles are created so no need to create an environment again and again for transform machine learning methods. At the end of this model, all outputs coming from different machine learning techniques plotting graph for compare between methods and analysis model.

This model based on supervised machine learning. The inputs taken from twitter and classified them into ternary classification. In this classification are divided into positive, negative and neutral. In this model taken labeled data from dataset and classified given reviews to ternary classification. This model also shown comparison between one method to another methods and provide result in terms of accuracy.

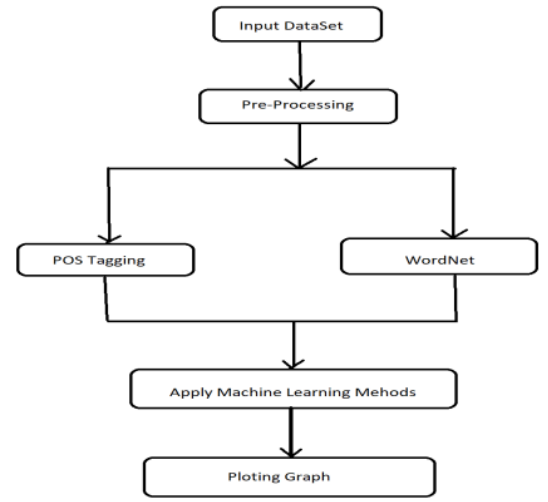

Fig. 2.Generic Architecture of NLP/Text Analytics

This main reason for taking tweets as data set because tweets have limitation as 140 characters. But this data set needs more preprocessing like hash tag '\#', at the rate '@' and re-tweet must be removed.

Published By:

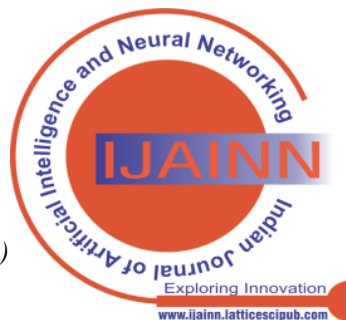




\section{Ternary Classification of Product Based Reviews: Survey, Open Issues and New Approach for Sentiment Analysis}

After cleaning data it is imported to extracted data from cleaned data set. Now this data set manually labeled with POS tag. This model used nltk tokenizer like sent_tokenize for sentence tokenization and word_tokenize for word tokenization then passes it for POS tagging. After labeling dataset, extract features from dataset then divided into training set and test set.

\section{COMPARISON WORK}

Throughout this survey clearly mentioned that all kind of work based on only document analysis with either text based or emotion based with limited symbols. Table -01 shown author with their methodology, classification, datasets and outcomes parameters

Table- I: Comparative Literature Survey

\begin{tabular}{|c|c|c|c|c|c|c|c|}
\hline SR.NO. & $\begin{array}{c}\text { AUTHOR } \\
\text { NAME/ } \\
\text { YEAR/ } \\
\text { PAPER } \\
\text { NUMBER }\end{array}$ & METHODOLOGY & DATASET & $\begin{array}{l}\text { EVARIOUS FIELD } \\
\text { OF SENTIMENT } \\
\text { ANALYSIS }\end{array}$ & $\begin{array}{l}\text { LABELS/ } \\
\text { CLASSES }\end{array}$ & LIMITAION & PARAMETERS \\
\hline 1 & $\begin{array}{l}\text { B. } \\
\text { Seetharamulu } \\
\text { et. al. (2020) }\end{array}$ & DL-SA & $\begin{array}{l}\text { Product } \\
\text { based } \\
\text { reviews on } \\
\text { amazon.com }\end{array}$ & $\begin{array}{l}\text { Emotion } \\
\text { recognition }\end{array}$ & $\begin{array}{l}\text { Positive } \\
\text { and } \\
\text { negative }\end{array}$ & $\begin{array}{l}37,126 \\
\text { reviews }\end{array}$ & $\begin{array}{l}\text { Accuracy based } \\
\text { on True positive } \\
\text { and true } \\
\text { negative }\end{array}$ \\
\hline 2 & $\begin{array}{l}\text { Azwa Abdul } \\
\text { Aziz et. al } \\
(2019)\end{array}$ & CA tree structure & $\begin{array}{l}\text { Amazon } \\
\text { dataset(book } \\
\text { kitchen, } \\
\text { electronic, } \\
\text { DVD) }\end{array}$ & Sentence-level & $\begin{array}{l}\text { Positive, } \\
\text { negative } \\
\text { and } \\
\text { neutral }\end{array}$ & $\begin{array}{l}3000 \\
\text { reviews }\end{array}$ & $\begin{array}{l}\text { TF(term } \\
\text { frequency)/ } \\
\text { percentage } \\
\text { method }\end{array}$ \\
\hline 3 & $\begin{array}{l}\text { Kartikkayini } \\
\text { T (2017) }\end{array}$ & Senti algorithm & $\begin{array}{l}\text { Product } \\
\text { based } \\
\text { reviews on } \\
\text { amazon.com }\end{array}$ & Sentence level & $\begin{array}{l}\text { Positive, } \\
\text { negative } \\
\text { and } \\
\text { neutral }\end{array}$ & $\begin{array}{l}100 \text { calls for } \\
\text { API }\end{array}$ & $\begin{array}{l}\text { Review ID, } \\
\text { reviewText }\end{array}$ \\
\hline 4 & $\begin{array}{l}\text { Ronen } \\
\text { Feldman } \\
\text { (2013) }\end{array}$ & $\begin{array}{l}\text { Comparative with } \\
\text { exiting methods }\end{array}$ & $\begin{array}{l}\text { Stocks } \\
\text { market data }\end{array}$ & All fields & $\begin{array}{l}\text { Positive, } \\
\text { negative } \\
\text { and } \\
\text { neutral }\end{array}$ & $\begin{array}{l}\text { Not worked } \\
\text { on incorrect } \\
\text { sentences }\end{array}$ & $\begin{array}{l}\text { stockTwits, } \\
\text { Ticker }\end{array}$ \\
\hline 5 & $\begin{array}{l}\text { K. Saranya } \\
\text { and S. } \\
\text { Jayanthy } \\
(2017)\end{array}$ & $\begin{array}{l}\text { Hybrid algorithms } \\
\text { (SVM+ } \\
\text { Navie-Bayes) }\end{array}$ & $\begin{array}{l}\text { Movies } \\
\text { reviews }\end{array}$ & Emoword & $\begin{array}{l}\text { Positive } \\
\text { and } \\
\text { negative }\end{array}$ & $\begin{array}{l}\text { Dataset } \\
\text { creation is } \\
\text { relatively } \\
\text { complex }\end{array}$ & $\begin{array}{l}\text { Accurate } \\
\text { classification, } \\
\text { accuracy of } \\
\text { binary } \\
\text { classification }\end{array}$ \\
\hline 6 & $\begin{array}{l}\text { Nadijim } \\
\text { Frechet et al. } \\
\text { (2019) }\end{array}$ & Text-analysis & $\begin{array}{l}50 \text { top } \\
\text { university } \\
\text { syllabus for } \\
\text { political } \\
\text { science }\end{array}$ & Indexing & $\begin{array}{l}\text { Score } \\
\text { scale }\end{array}$ & $\begin{array}{l}1000 \text { unique } \\
\text { sources }\end{array}$ & $\begin{array}{l}\text { Predictive } \\
\text { accuracy }\end{array}$ \\
\hline 7 & $\begin{array}{l}\text { K. Sudheer } \\
\text { and Dr. B } \\
\text { Valarmathi } \\
\text { (2018) }\end{array}$ & $\begin{array}{l}\text { Document } \\
\text { Frequency(DF) }\end{array}$ & $\begin{array}{l}\text { Tweets from } \\
\text { twitter }\end{array}$ & Sentence level & $\begin{array}{l}\text { Positive, } \\
\text { negative } \\
\text { and } \\
\text { neutral }\end{array}$ & $\begin{array}{l}\text { Approximate } \\
50000\end{array}$ & $\begin{array}{l}\text { Quality and } \\
\text { timely delivery }\end{array}$ \\
\hline 8 & $\begin{array}{l}\text { Lijuan Huang } \\
\text { et al (2019) }\end{array}$ & PTSM & $\begin{array}{l}\text { Daily movies } \\
\text { online } \\
\text { reviews }\end{array}$ & $\begin{array}{l}\text { Document-level and } \\
\text { corpus }\end{array}$ & $\begin{array}{l}\text { Positive } \\
\text { and } \\
\text { negative }\end{array}$ & $\begin{array}{l}13417 \\
\text { reviews }\end{array}$ & $\begin{array}{l}\text { Prediction } \\
\text { accuracy }\end{array}$ \\
\hline
\end{tabular}

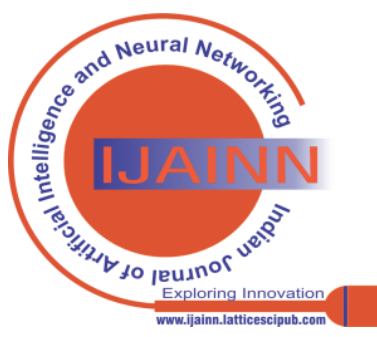




\begin{tabular}{|c|c|c|c|c|c|c|c|}
\hline 9 & $\begin{array}{l}\text { Abinash } \\
\text { Tripathy et } \\
\text { al. (2017) }\end{array}$ & $\begin{array}{ll}\begin{array}{l}\text { Hybrid of } \\
\text { and }\end{array} & \text { SVM } \\
\text { algorithm } & \end{array}$ & $\begin{array}{l}\text { Two movie } \\
\text { reviews } \\
\text { dataset } \\
\text { IMDb } \\
\text { dataset and } \\
\text { polarity } \\
\text { dataset }\end{array}$ & $\begin{array}{l}\text { CountVectorization } \\
\text { frequency-inverse } \\
\text { document frequency } \\
\text { and word2vec }\end{array}$ & $\begin{array}{l}\text { Positive } \\
\text { and } \\
\text { negative }\end{array}$ & $\begin{array}{l}\text { IMDb } \\
12500,1000 \\
\text { positive } \\
\text { polarity and } \\
1000 \\
\text { negative } \\
\text { polarity }\end{array}$ & $\begin{array}{l}\text { Precision, recall } \\
\text { and accuracy }\end{array}$ \\
\hline 10 & $\begin{array}{l}\text { Jaspreet } \\
\text { Singh and } \\
\text { Gurvinder } \\
\text { Singh (2018) }\end{array}$ & SVM and GBDT & $\begin{array}{l}\text { Reviews } \\
\text { based on } \\
\text { Amazon web } \\
\text { services }\end{array}$ & $\begin{array}{l}\text { Post processing } \\
\text { using UTF-8 }\end{array}$ & $\begin{array}{l}\text { Positive } \\
\text { and } \\
\text { negative }\end{array}$ & $\begin{array}{l}\text { Not clearly } \\
\text { define } \\
\text { response } \\
\text { time, latency } \\
\text { and } \\
\text { execution } \\
\text { time of the } \\
\text { model }\end{array}$ & QoS \\
\hline 11 & $\begin{array}{l}\text { Jeong Woong } \\
\text { Sohn and Jin } \\
\text { Ki Kim } \\
(2020)\end{array}$ & $\begin{array}{l}\text { SNS functions like } \\
\text { content shearing, } \\
\text { awareness }\end{array}$ & $\begin{array}{l}\text { Scoical } \\
\text { network sites } \\
\text { in korea from } \\
\text { Groupon, } \\
\text { google and } \\
\text { amazon }\end{array}$ & $\begin{array}{l}\text { Social commerce } \\
\text { attributes }\end{array}$ & $\begin{array}{l}\text { Purchase } \\
\text { intention }\end{array}$ & $\begin{array}{l}\text { Model } \\
\text { dependent } \\
\text { on factor } \\
\text { analysis and } \\
\text { its time } \\
\text { consuming }\end{array}$ & $\begin{array}{l}\text { Price, quality, } \\
\text { variety, } \\
\text { reliability, } \\
\text { customer } \\
\text { convenience }\end{array}$ \\
\hline 12 & $\begin{array}{l}\text { Valerio } \\
\text { Basile et al. } \\
\text { (2018) }\end{array}$ & SENTIPOLC & $\begin{array}{l}\text { EVALITA } \\
\text { evaluation } \\
\text { campaign }\end{array}$ & $\begin{array}{lr}\text { CNN } & \text { with } \\
\text { SwissCheese } & \text { and } \\
\text { Unitor } & \end{array}$ & $\begin{array}{l}\text { Ironic, } \\
\text { subjective } \\
\text { and } \\
\text { polarity }\end{array}$ & 2000 & $\begin{array}{l}\text { Less error ( with } \\
\text { respect to wrong } \\
\text { label) }\end{array}$ \\
\hline 13 & $\begin{array}{l}\text { Coruelius } \\
\text { Puschmann } \\
\text { and Alison } \\
\text { Powell } \\
\text { (2018) }\end{array}$ & $\begin{array}{l}\text { Linguistic Inquiry } \\
\text { and Word Count } \\
\text { (LIWC) }\end{array}$ & $\begin{array}{l}\text { News papers } \\
\text { articles from } \\
\text { US, UK and } \\
\text { Germany }\end{array}$ & $\begin{array}{l}\text { Document level } \\
\text { sentiment }\end{array}$ & $\begin{array}{l}\text { Positive, } \\
\text { negative } \\
\text { and } \\
\text { neutral }\end{array}$ & 1062 articles & $\begin{array}{l}\text { Qualities and } \\
\text { capacities }\end{array}$ \\
\hline 14 & 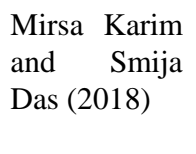 & LDA Analysis & $\begin{array}{l}\text { Movies } \\
\text { reviews from } \\
\text { cornell }\end{array}$ & $\begin{array}{l}\text { Sentiment VADER } \\
\text { abd Senti word net }\end{array}$ & $\begin{array}{l}\text { Positive, } \\
\text { negative } \\
\text { and } \\
\text { neutral }\end{array}$ & $\begin{array}{l}\text { Not } \\
\text { normalized } \\
\text { less accurate } \\
\text { data }\end{array}$ & Accuracy \\
\hline
\end{tabular}

\section{CONCLUSION}

Before buying of any product customers believe to find all kind of reviews related to it before making their mind. These reviews based analyses provide a very big field to research area. Through reviews seller can predict customer opinion. Document based Sentiment analysis provide a accurate prediction of customer reviews. Compile all the open issues and challenges of document based sentiment analysis that define binary or multiclass classification. This paper is based on document based sentiment analysis with ternary classification. Which is provide three concrete classes: positive, negative and neutral.

\section{REFERENCES}

1. B. Seetharamulu, B. Naresh Kumar Reddy and K. Bramha Naidu, "Deep Learning for Sentiment Analysis Based on Customer Reviews". IEEE $11^{\text {th }}$ ICCCNT, 2020[ ICFAI foundation for higher Education, Hyderabad, India]. [CrossRef]

2. Azwa Abdul Aziz, Andrew Starkey, Elissa Nadia Madi, "Prediction supervised Machine Learning Performances for sentiment analysis using contextual based approaches", IEEE Access, 2019. [CrossRef]

3. Karthikkayini T, N. K. Srinath. "Comparative polarity analysis on Amazon product reviews using existing machine learning algorithms". IEEE International Conference, 2017. [CrossRef]

4. Ronen Feldman. "Techniques and Application for Sentiment Analysis". ACM, vol. 56, no. 4, 2013. [CrossRef]
5. K. Saranya and Dr. S. Jayanthy, "Onto-based sentiment classification using Machine Learning Techniques”, ICIIECS, 2017. [CrossRef]

6. Nadjim Frechet, Justin Savoie and Yannick Dufresne, "Analysis of Text-Analysis Syllabi: Buliding a Text-Analysis Syllabus Using Scaling”, American Political Science Association, 2019. [CrossRef]

7. K. Sudheer, Dr. B Valarmathi, SITE, "Real Time Sentiment Analysis of E-Commerce Websites using Machine Learning Algorithms". IJMET, Volume 9, Issue 2, February 2018, pp 180-193.

8. Lijuan huang, Zixin Dou, Yongjun Hu and Raoyi Huang, "Textual Analysis for Online Reviews: A Polymerization Topic Sentiment Model”. IEEE Access, Volume 7, 25 July 2019, pp 91940-91945. [CrossRef]

9. Abinash Tripathy, Abhishek Anand and Santanu Kumar Rath, "Document-level sentiment classification using hybrid machine learning approach", Springer-Verlag London 10 May 2017. [CrossRef]

10. Jaspreet Singh and Gurvinder Singh,“ Sentiment Analysis of Social Media Reviews using QOS Parameterization”. ICSCCC, 2018

11. Jeong Woong Sohn, Jin Ki Kim, "Factors that influence purchase intentions in social commerce". ScienceDirect, 26 August, 2020.

12. Valerio Basile, Nicole Novielli, Danilo Croce, Francesco Barbieri, Malvina Nissim and Viviana Patti, "Sentiment Polarity Classification at EVALITA: Lessons Learned and Open Challenges". IEEE, 2018.

13. Cornelius Puschmann and Alison Powell, "Turning Words Into Consumer Preferences: How Sentiment Analysis Is Framed in Research and the News Media". SAGE, Social Media + Society July-September 2018. [CrossRef] 
14. Mirsa Karim and Smija Das, "Sentiment Analysis on Textual Reviews". IOP publishing, conf: Series Material Science and Engineering 396, 2018. [CrossRef]

15. Jin Zhou and Jun-min Ye, "Sentiment analysis in education research: a review of journal publications". Interactive learning environments. 12 September, 2020. [CrossRef]

\section{AUTHORS PROFILE}

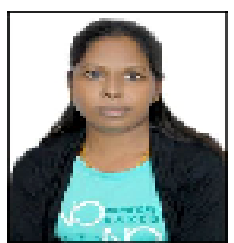

Sushila Sonare was born in India on August 22, 1982. She is research scholar in LNCTU, Bhopal. She completed her M.Tech. in Computer Science and Engineering from NIT Calicut, Karala, in 209, and B.E in Computer Science and Engineering from Jabalpur Engineering College, in 2006. She has working experience as $10+$ years including industrial and teaching. Her research interest fields are machine learning, deep learning, data mining, NLP, Data Analysis and computer network.

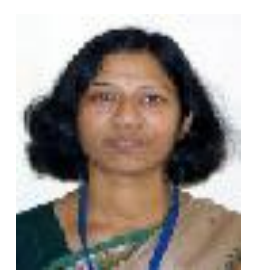

Dr. Megha Kamble was born in India on April 6, 1976. She received the Engineering degree, B.E. in Computer Science and engineering from Govt. engineering college, Aurangabad (Maharashtra) in 1996 and post graduate degree M.Tech. in Computer Science and Engineering and the Ph.D. degree in Information Technology from the Rajiv Gandhi State Technical University, Bhopal, Madhya Pradesh, India, in 2007 and 2017, respectively. She is currently a Professor in the Department of Computer Science and Engineering, at Lakshmi Narain College of Technology, Madhya Pradesh, India, and has been the Head of Department and R\&D cell convener from 2008 to 2015 in reputed institution in M.P. India. She possesses 21 years of experience including teaching, industrial and research work She was annual member of CSI from 2011 to 2014 and member of IAENG, life member of SSIRT. She has published more than 30 scientific papers in International and National reputed Journals and conference proceedings in the field of mobile computing, soft computing, image processing, and wireless networks. Her current research interests include aging Multi gent System, Deep learning, Machine Learning, Soft Computing, Cellular Network, Channel Allocation, Software Engineering, Computer Graphics, Data analytics, IoT, NLP. She had been student project mentors under IEDC, and Smart India Hackathon, Govt of India initiative. She has been invited for expert talks for DST, Govt of India funded FDPs on Big data and AI in Bhopal, India. She has completed TEQIP funded CRS project on Solar Irradiance prediction using applied machine learning.

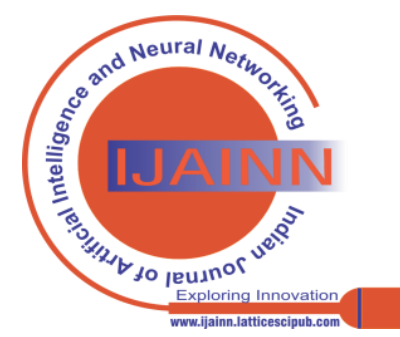

